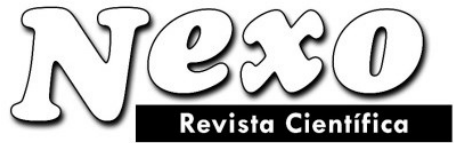

\title{
EFECTO DE LA RELACIÓN DE ESBELTEZ EN LA CINEMÁTICA DEL FLUJO ROTATORIO CON FRONTERAS RÍGIDAS
}

\section{EFFECT OF SLENDERNESS RATIO IN THE KINEMATIC THE ROTATING FLOW WITH RIGID FRONTIERS}

\author{
A. Lizardi*, H. Terres, R. López, M. Vaca, J. Flores, S. Chávez \\ Universidad Autónoma Metropolitana-Azcapotzalco; \\ Departamento de Energía, Área de Termofluidos. \\ Av. San Pablo 180, Col. Reynosa Tamaulipas; Del. Azcapotzalco, C.P. 02200, México D. F. \\ Tel.: 52(55)5318-9060 Fax: 52(55)5318-9058 \\ E-Mai1*: arlr@correo.azc.uam.mx
}

(recibido/received: 24-Julio-2014; aceptado/accepted: 4-Junio-2015)

\begin{abstract}
RESUMEN
En este trabajo se analizan las componentes de velocidad radial (u), azimutal (v), los flujos volumétricos primario $(\mathrm{Qp})$, secundario $(\mathrm{Qs})$ y el coeficiente de par $(\mathrm{Cm})$, que se generan en el interior de un cilindro cerrado que tiene la tapa giratoria y el fondo y envolvente sin movimiento. Los resultados se presentan mediante gráficas en las que se analizan dichas propiedades, en régimen laminar, para un número de Reynolds de 3x104 y para un factor de forma (alto/radio) del recipiente de 1.0, 1.25, 1.5, 1.75 y 2.0. Tomando como referencia la relación geométrica de 1.0 y comparándolo con los otros factores de forma, se encontró que: la componente de velocidad radial (u) se incrementó en su valor máximo positivo en un $14.02 \%$; la componente de velocidad tangencial (v) mantuvo los mismos valores máximos en todas las relaciones geométricas; el flujo volumétrico primario aumentó en un $193.25 \%$; el flujo volumétrico secundario se incrementó en un $236.48 \%$; y el coeficiente de par aumentó en un $94.41 \%$. Los resultados anteriores revelan que al modificar la relación de esbeltez del sistema las propiedades cinemáticas estudiadas no siguen un patrón de variación homogéneo y que dependerá del interés que se tenga en alguna de ellas para analizar su comportamiento.
\end{abstract}

Palabras claves: Flujo rotatorio; relación de esbeltez; flujo volumétrico; coeficiente de par.

\begin{abstract}
In this work we analyse the components of the radial velocity $(\mathrm{u})$, azimuthal (v), primary and secondary volumetric flows, $(\mathrm{Qp})$, and $(\mathrm{Qs})$, and the torque coefficient $(\mathrm{Cm})$, all of which are produced inside a closed cylinder which has a rotary lid and the bottom and wraparound surface are static. The results are graphically presented, considering a Reynolds number laminar of $3 \times 10^{4}$ and for container form factors (height/radius) of 1.0, 1.25, 1.5, 1.75, and 2.0. Starting from a geometric ratio of 1.0 and comparing it to the rest of form factors, it was observed that the radial velocity component $(\mathrm{u})$ increased its maximum positive value in a $14.02 \%$; the tangential velocity component kept the same maximum values in all the geometric ratios; the primary volumetric flow increased by $193.25 \%$; the secondary volumetric flow increased by $236.48 \%$; and the torque coefficient also increased by $94.41 \%$. These results imply that in modifying the slenderness ratio of the system, the studied kinematic properties do not follow an homogeneous variation pattern and depending on the concern of each of them, its behaviour should be analysed in detail.
\end{abstract}

Key words: Rotating flow; slenderness ratio; volumetric flow; torque coefficient. 


\section{INTRODUCCIÓN}

La rotación de un fluido que se mueve alrededor de un eje vertical puede ser de dos formas; movimiento como vórtice forzado y movimiento como vórtice libre o torbellino potencial. En el primer caso, cada partícula de fluido tiene la misma velocidad angular, los esfuerzos cortantes son nulos, la única aceleración que ocurre se dirige radialmente hacia el eje de rotación, y se forma una superficie libre en forma de paraboloide de revolución; en el segundo caso, cada partícula se mueve con una velocidad que varía inversamente a la distancia desde el centro de rotación, el flujo generado es irrotacional, el cambio de momento angular es cero, y se forma una superficie libre en forma de hiperboloide de revolución. En particular el flujo rotatorio es atractivo desde el punto de vista del análisis teórico ya que las ecuaciones que lo caracterizan son considerablemente complejas y no han sido resueltas, hasta la fecha, por métodos analíticos. En este trabajo, la ecuación de Navier-Stokes, ecuación diferencial parcial no lineal y elíptica, que describe el flujo rotatorio se aborda desde el punto de vista de la dinámica de fluidos computacional a través del método numérico de diferencias finitas obteniéndose soluciones particulares para el caso específico de un recipiente cilíndrico cerrado.

Al paso del tiempo, algunos de los investigadores que han abordado este problema son: Pao H-P (1972) hizo un cálculo numérico de un fluido viscoso incompresible confinado en una cámara cilíndrica vertical con un disco rotando en la parte superior, a una velocidad angular constante, y con el fondo y paredes laterales fijas. Se resolvieron las ecuaciones de Navier-Stokes para flujo permanente y para valores del número de Reynolds menores a 400. Se encontró que para números de Reynolds bajos $(R e=10)$ las ecuaciones gobernantes son esencialmente lineales y la solución numérica se aproxima muy bien a la solución analítica. Para números de Reynolds altos el flujo en las fronteras se intensifica. Los detalles de los campos de flujo se comparan con trabajos previos. Bertelá M. y Gori F. (1982) analizaron el flujo permanente y transitorio en una cámara cilíndrica con su cubierta rotando. Se analizaron dos números de Reynolds (100 y 1000) y tres relaciones geométricas $(0.5,1.0$ y 2.0$)$. Se describe la estructura de los capos de velocidad y de presión en el sistema. También se analizan los valores del flujo volumétrico primario, secundario y coeficiente de par, para los seis casos analizados. Entre los resultados transitorios se encontraron algunas diferencias en el comportamiento del flujo para $\mathrm{Re}=100 \mathrm{y} \mathrm{Re}=1000$. En el primer caso el transporte de momento angular fue esencialmente difusivo; en el segundo caso esta cantidad es influenciada por la acción centrífuga del disco rotatorio encontrándose una mezcla de carácter difusivo y convectivo. Lang E. et al. (1994) estudiaron numéricamente el flujo estacionario en régimen laminar en un cilindro fijo con un disco rotando en la tapa superior. Las ecuaciones gobernantes en coordenadas cilíndricas fueron resueltas por el método ADI (alternating-direction implicit). Los rangos investigados fueron: número de Reynolds 1 a 105; relación geométrica 0.02 a 3; huelgo entre el extremo del disco y la pared del cilindro 0.1 y $10 \%$ del radio del recipiente. El trabajo resalta el análisis del coeficiente de par y de los flujos volumétricos primario y secundario. Los resultados mostraron que dichas cantidades son dependientes del número de Reynolds y de la relación geométrica. El coeficiente de par fue dependiente del número de Reynolds y del huelgo, mientras el flujo volumétrico lo fue solamente del huelgo. Khalili A. y Adabala R. R. (1995) proveyeron una solución numérica para un flujo laminar inducido por un disco rotatorio situado asimétricamente respecto a la altura dentro de un cilindro. Presentaron el análisis de las líneas de corriente y componentes de velocidad tangencial así como el coeficiente de par para los distintos posicionamientos del disco, para una relación geométrica de 1 a 2 y un rango de números de Reynolds de 1 a 5000. Lizardi R. A, et al. (2007) presentaron la comparación experimental y numérica del flujo generado en el interior de un cilindro vertical con tapa giratoria y el fondo y paredes fijas. El desarrollo experimental se realizó con Velocimetría de Imágenes de Partículas (PIV), con el cual se obtuvieron los campos de las componentes de velocidad radial, tangencial y axial. Se resolvieron numéricamente las ecuaciones de continuidad y de conservación de la cantidad de movimiento para un flujo permanente con fluido viscoso e incompresible confinado en un cilindro vertical cerrado, aplicando el método de diferencias finitas. La relación del depósito alto/radio fue igual a la unidad y el número de Reynolds fue de 5x104. Yu P., et al. (2007) estudiaron numéricamente el flujo en una cámara cilíndrica cerrada con un disco giratorio en el fondo y cuyo radio es menor al de la cámara. El comportamiento del flujo se investigó para una amplia gama de parámetros. Se analizaron, en el plano meridional, las líneas de corriente, momento angular y vorticidad, para diferentes Reynolds (1000, 1500 y 2000), relación geométrica $\mathrm{H} / \mathrm{R}(1.5)$ y relación de radios $\mathrm{R} / \mathrm{rd}(1.5,1.8,2.0,2.2,2.6,3.0$,

Nexo Revista Científica Vol. 28, No. 01, pp. 01-14/Junio 2015 
5.0). Sturzenegger J. C., et al. (2012) estudiaron el flujo axisimétrico dentro en un recipiente cilíndrico con una varilla a lo largo de su eje de simetría. El flujo se produce por la rotación de uno de los extremos del cilindro, de ambos extremos, o de la pared lateral. Se presentan expresiones analíticas (para números de Reynolds bajos) del campo de velocidad azimutal, extendiendo la solución para el caso sin varilla.

El objetivo de este trabajo es analizar las componentes de velocidad radial (u), azimutal (v), los flujos volumétricos primario $(\mathrm{Qp})$, secundario $(\mathrm{Qs})$ y coeficiente de par $(\mathrm{Cm})$ que se generan en el interior de un cilindro cerrado que tiene la tapa superior giratoria y el fondo y envolvente sin movimiento. Los resultados se presentan mediante gráficas comparativas, en régimen laminar para un número de Reynolds de 3x104 y para un factor de forma (alto/radio) del recipiente de 1.0, 1.25, 1.5, 1.75 y 2.0.

\section{MODELO FÍSICO}

El modelo analizado consta de un cilindro de radio $\mathrm{E}$ y altura $\mathrm{H}$, el cual tiene un impulsor radial en la parte superior, y su envolvente y fondo se encuentran fijos. En el interior del cilindro se tiene un fluido newtoniano, viscoso e incompresible. El impulsor se mueve a una velocidad angular constante $\Omega$ y se estudian cinco factores de forma (H/E): 1.0, 1.25, 1.5, 1.75 y 2.0 .
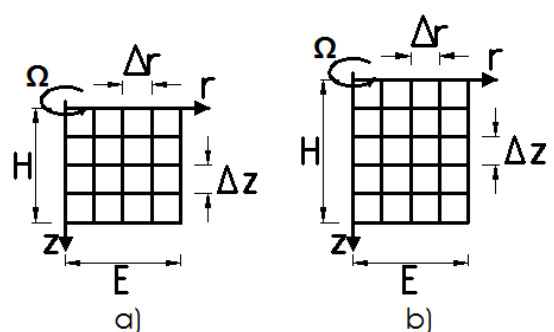

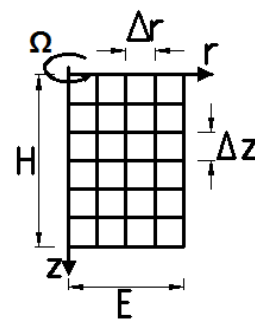

c)

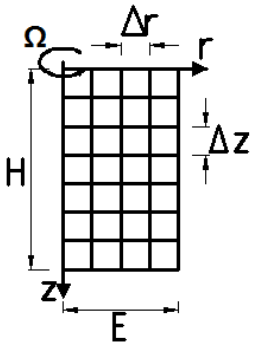

d)

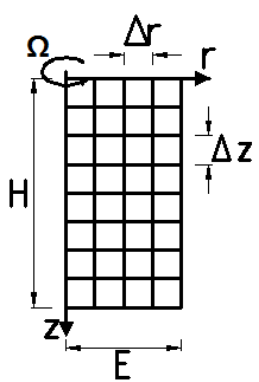

e)

Fig. 1 Modelo físico del sistema a analizar para un factor de forma $(\mathrm{H} / \mathrm{E})$ de: a) 1.0 , b) 1.25 , c) 1.5 , d) 1.75 , e) 2.0

\section{PLANTEAMIENTO MATEMÁTICO}

De acuerdo a la geometría descrita, el planteamiento matemático se expresa a través de las ecuaciones de continuidad (1) y cantidad de movimiento (2), (3) y (4), en coordenadas polares cilíndricas (R, $\Phi, Z$ ). Para un fluido viscoso, incompresible, de propiedades físicas constantes, en flujo permanente y axisimétrico, se expresan como (Landau y Lisfshits, 1982)

$$
\begin{gathered}
\frac{\partial \mathrm{U}}{\partial \mathrm{R}}+\frac{\mathrm{U}}{\mathrm{R}}+\frac{\partial \mathrm{W}}{\partial \mathrm{Z}}=0 \\
\mathrm{U} \frac{\partial \mathrm{U}}{\partial \mathrm{R}}-\frac{\mathrm{V}^{2}}{\mathrm{R}}+\mathrm{W} \frac{\partial \mathrm{U}}{\partial \mathrm{Z}}=-\frac{1}{\rho} \frac{\partial \mathrm{P}}{\partial \mathrm{R}}+\mathrm{v}\left[\frac{\partial^{2} \mathrm{U}}{\partial \mathrm{R}^{2}}+\frac{1}{\mathrm{R}} \frac{\partial \mathrm{U}}{\partial \mathrm{R}}-\frac{\mathrm{U}}{\mathrm{R}^{2}}+\frac{\partial^{2} \mathrm{U}}{\partial \mathrm{Z}^{2}}\right] \\
\mathrm{U} \frac{\partial \mathrm{V}}{\partial \mathrm{R}}+\frac{\mathrm{U} \mathrm{V}}{\mathrm{R}}+\mathrm{W} \frac{\partial \mathrm{V}}{\partial \mathrm{Z}}=v\left[\frac{\partial^{2} \mathrm{~V}}{\partial \mathrm{R}^{2}}+\frac{1}{\mathrm{R}} \frac{\partial \mathrm{V}}{\partial \mathrm{R}}-\frac{\mathrm{V}}{\mathrm{R}^{2}}+\frac{\partial^{2} \mathrm{~V}}{\partial \mathrm{Z}^{2}}\right] \\
\mathrm{U} \frac{\partial \mathrm{W}}{\partial \mathrm{R}}+\mathrm{W} \frac{\partial \mathrm{W}}{\partial \mathrm{Z}}=-\frac{1}{\rho} \frac{\partial \mathrm{P}}{\partial \mathrm{Z}}+v\left[\frac{\partial^{2} \mathrm{~W}}{\partial \mathrm{R}^{2}}+\frac{1}{\mathrm{R}} \frac{\partial \mathrm{W}}{\partial \mathrm{R}}+\frac{\partial^{2} \mathrm{~W}}{\partial \mathrm{Z}^{2}}\right]-\mathrm{g}
\end{gathered}
$$

Este conjunto de ecuaciones dimensionales tiene como incógnitas las componentes de velocidad radial 
(U), azimutal (V), axial (W) y la presión (P). La presión depende del valor de las componentes de velocidad en cualquier punto del sistema. Para reducir este término se hace una transformación de las ecuaciones de continuidad y cantidad de movimiento radial, tangencial y axial, en función de la circulación $\left({ }^{\gamma}\right)$, vorticidad $(\varsigma)$, y función corriente meridional $(\psi)$. La forma adimensional resultante es (Pao H-P, 1972)

$$
\begin{gathered}
\mathrm{u} \frac{\partial \Gamma}{\partial \mathrm{r}}+\mathrm{w} \frac{\partial \Gamma}{\partial \mathrm{z}}=\frac{1}{\operatorname{Re}}\left[\nabla^{2} \Gamma-\frac{2}{\mathrm{r}} \frac{\partial \Gamma}{\partial \mathrm{r}}\right] \\
\mathrm{u} \frac{\partial \xi}{\partial \mathrm{r}}+\mathrm{w} \frac{\partial \xi}{\partial \mathrm{z}}-\frac{\mathrm{u} \xi}{\mathrm{r}}-\frac{1}{\mathrm{r}^{3}} \frac{\partial\left(\Gamma^{2}\right)}{\partial \mathrm{z}}= \\
\frac{1}{\operatorname{Re}}\left[\nabla^{2} \xi-\frac{\xi}{\mathrm{r}^{2}}\right] \\
\nabla^{2} \Psi-\frac{2}{\mathrm{r}} \frac{\partial \Psi}{\partial \mathrm{r}}=\mathrm{r} \xi
\end{gathered}
$$

En las ecuaciones anteriores se han definido algunos parámetros que relacionan a las variables adimensionales (r, z, u, v, w, $\Psi, \Gamma, \xi$ ) con las dimensionales (R, Z, U, V, W, $\left.\psi,{ }^{\gamma}, \varsigma\right)$. Éstos se muestran en las ecuaciones (8) y (9)

$$
\begin{gathered}
\frac{\mathrm{R}}{\mathrm{E}}=\mathrm{r}, \frac{\mathrm{Z}}{\mathrm{E}}=\mathrm{z}, \frac{\mathrm{U}}{\Omega \mathrm{E}}=\mathrm{u}, \frac{\mathrm{V}}{\Omega \mathrm{E}}=\mathrm{v}, \frac{\mathrm{W}}{\Omega \mathrm{E}}=\mathrm{w}, \frac{\psi}{\Omega \mathrm{E}^{3}}=\Psi, \frac{\gamma}{\Omega \mathrm{E}^{2}}=\Gamma, \frac{\varsigma}{\Omega}=\xi, \\
\operatorname{Re}=\frac{\Omega \mathrm{E}^{2}}{v}
\end{gathered}
$$

con

$$
\begin{aligned}
& \gamma=2 \pi \mathrm{VR}, \quad \varsigma=\frac{\partial \mathrm{U}}{\partial \mathrm{Z}}-\frac{\partial \mathrm{W}}{\partial \mathrm{R}}, \\
& \mathrm{U}=\frac{1}{\mathrm{R}} \frac{\partial \psi}{\partial \mathrm{Z}}, \quad \mathrm{W}=-\frac{1}{\mathrm{R}} \frac{\partial \psi}{\partial \mathrm{R}}
\end{aligned}
$$

En estas ecuaciones E es el radio del cilindro, $\Omega$ es la velocidad angular del disco impulsor y Re es el número de Reynolds definido para sistemas cilíndricos. Las condiciones de frontera apropiadas para este caso son (Gerber N., 1975)

\begin{tabular}{|l|l|l|l|l|}
\hline $\mathrm{r}=0$, & $\Psi=0$, & $\Gamma=0$, & $\xi=0$ & $\xi=\frac{1}{\mathrm{r}} \frac{\partial^{2} \Psi}{\partial \mathrm{r}^{2}}$ \\
\hline $\mathrm{r}=\mathrm{E}$, & $\Psi=0$, & $\Gamma=0$, & $\xi=\frac{1}{\mathrm{r}} \frac{\partial^{2} \Psi}{\partial \mathrm{z}^{2}}$ & \\
\hline $\mathrm{z}=0$, & $\Psi=0$, & $\Gamma=\mathrm{r}^{2}$, & $\xi=\frac{1}{\mathrm{r}} \frac{\partial^{2} \Psi}{\partial \mathrm{z}^{2}}$ & \\
\hline $\mathrm{z}=\mathrm{H}$, & $\Psi=0$, & $\Gamma=0$, & $(10)$ \\
\hline
\end{tabular}




\section{SOLUCIÓN NUMÉRICA}

Para resolver las ecuaciones de transporte de la circulación (5), de la vorticidad tangencial (6) y de la función corriente meridional (7), junto con las condiciones de frontera apropiadas (10), es necesario transformarlas a un espacio discreto, que es el espacio manejado por las computadoras, es decir, se transforman las ecuaciones en expresiones en forma de diferencias finitas. Estas ecuaciones fueron obtenidas a partir de las formas básicas, tal como diferencias hacia adelante, hacia atrás, y central de las derivadas. En la Fig. 1 se representa la malla en el plano (r, z) para flujo axisimétrico y para los cinco sistemas a analizar. Las ecuaciones en diferencias finitas que gobiernan el movimiento del fluido son (Lizardi R. A., et al., 2010), (Lizardi R. A, et al. 2013)

$$
\begin{aligned}
& \Psi_{\mathrm{i}, \mathrm{j}}=\frac{1}{2\left[1+\left(\frac{\Delta \mathrm{r}}{\Delta \mathrm{z}}\right)^{2}\right]}\left\{\Psi_{\mathrm{i}+1, \mathrm{j}}\left[1-\frac{1}{2 \mathrm{i}}\right]+\Psi_{\mathrm{i}-1, \mathrm{j}}\left[1+\frac{1}{2 \mathrm{i}}\right]+\left(\frac{\Delta \mathrm{r}}{\Delta \mathrm{z}}\right)^{2} \Psi_{\mathrm{i}, \mathrm{j}+1}+\right. \\
& \left.\left(\frac{\Delta r}{\Delta z}\right)^{2} \Psi_{i, j-1}-i(\Delta r)^{3} \xi_{i, j}\right\} \\
& \Gamma_{\mathrm{i}, \mathrm{j}}=\frac{1}{2\left[1+\left(\frac{\Delta \mathrm{r}}{\Delta \mathrm{z}}\right)^{2}\right]}\left\{\Gamma_{\mathrm{i}+1, \mathrm{j}}\left[\left(1-\frac{1}{2 \mathrm{i}}\right)-\frac{\mathrm{Re}}{4 \mathrm{i} \Delta \mathrm{z}}\left(\Psi_{\mathrm{i}, \mathrm{j}+1}-\Psi_{\mathrm{i}, \mathrm{j}-1}\right)\right]+\right. \\
& \Gamma_{\mathrm{i}-1, \mathrm{j}}\left[\left(1+\frac{1}{2 \mathrm{i}}\right)+\frac{\operatorname{Re}}{4 \mathrm{i} \Delta \mathrm{z}}\left(\Psi_{\mathrm{i}, \mathrm{j}+1}-\Psi_{\mathrm{i}, \mathrm{j}-1}\right)\right]+\Gamma_{\mathrm{i}, \mathrm{j}+1}\left[\left(\frac{\Delta \mathrm{r}}{\Delta \mathrm{z}}\right)^{2}+\frac{\operatorname{Re}}{4 \mathrm{i} \Delta \mathrm{z}}\left(\Psi_{\mathrm{i}+1, \mathrm{j}}-\Psi_{\mathrm{i}-1, \mathrm{j}}\right)\right]+ \\
& \left.\Gamma_{\mathrm{i}, \mathrm{j}-1}\left[\left(\frac{\Delta \mathrm{r}}{\Delta \mathrm{z}}\right)^{2}-\frac{\operatorname{Re}}{4 \mathrm{i} \Delta \mathrm{z}}\left(\Psi_{\mathrm{i}+1, \mathrm{j}}-\Psi_{\mathrm{i}-1, \mathrm{j}}\right)\right]\right\} \\
& \xi_{i, j}=i^{2}\left\{\xi_{i+1, j}\left[\left(1+\frac{1}{2 i}\right)-\frac{R e}{4 i \Delta z}\left(\Psi_{i, j+1}-\Psi_{i, j-1}\right)\right]+\xi_{i-1, j}\left[\left(1-\frac{1}{2 i}\right)+\frac{R e}{4 i \Delta z}\left(\Psi_{i, j+1}-\Psi_{i, j-1}\right)\right]+\right. \\
& \xi_{i, j+1}\left[\left(\frac{\Delta r}{\Delta z}\right)^{2}+\frac{R e}{4 i \Delta z}\left(\Psi_{i+1, j}-\Psi_{i-1, j}\right)\right]+ \\
& \xi_{i, j-1}\left[\left(\frac{\Delta r}{\Delta z}\right)^{2}-\frac{\operatorname{Re}}{4 \mathrm{i} \Delta \mathrm{z}}\left(\Psi_{\mathrm{i}+1, \mathrm{j}}-\Psi_{\mathrm{i}-1, \mathrm{j}}\right)\right]+
\end{aligned}
$$$$
\left.\left[\frac{\operatorname{Re} \Gamma_{\mathrm{i}, \mathrm{j}}}{\mathrm{i}^{3}(\Delta \mathrm{r})(\Delta \mathrm{z})}\left(\Gamma_{\mathrm{i}, \mathrm{j}+1}-\Gamma_{\mathrm{i}, \mathrm{j}-1}\right)\right]\right\} \quad\left[2 \mathrm{i}^{2}+2 \mathrm{i}^{2}\left(\frac{\Delta \mathrm{r}}{\Delta \mathrm{z}}\right)^{2}+\right.
$$$$
\left.1-\frac{\operatorname{Re}}{2 \Delta \mathrm{z}}\left(\Psi_{\mathrm{i}, j+1}-\Psi_{\mathrm{i}, \mathrm{j}-1}\right)\right]^{-1}
$$

Para determinar la distribución de la función corriente meridional $(\Psi)$, circulación $(\Gamma) \mathrm{y}$ vorticidad tangencial $(\xi)$, en el interior del sistema, se generó un programa de cómputo en lenguaje $\mathrm{C}++$. El programa realiza el mallado en el plano (r, z), aplica las ecuaciones (11), (12) y (13) a cada nodo interno del sistema y las ecuaciones (10), previamente discretizadas, a cada nodo ubicado en la frontera del mismo. El programa solicita el valor del número de Reynolds, las dimensiones verticales y radiales del cilindro, la densidad de la malla y un valor inicial de $\xi(\mathrm{i}, \mathrm{j})$. Posteriormente resuelve el sistema de ecuaciones en forma iterativa hasta encontrar la convergencia. El criterio de convergencia utilizado es 
del tipo de error relativo, es decir, $m a ́ x\left[\frac{\Psi^{\sigma+1}-\Psi^{\sigma}}{\Psi^{\sigma}}\right]<\varepsilon$. El orden de precisión de $\varepsilon$, en este trabajo, fue de 0.0001. Según Lang E., et al., (1994), el tamaño de la malla recomendado para Reynolds menores a 1000 es de 81 x81, y para Reynolds menores a $10^{6}$ se han empleado mallas de $601 \times 601$. En este trabajo se utilizó una malla de 650x650, encontrando convergencia en los resultados y carencia de discontinuidades. La computadora utilizada fue una Dell Studio XPS, Intel(R) Core (TM) i7 CPU, 930 (a) $2.80 \mathrm{GHz}$ y el tiempo de convergencia para resolver el sistema de ecuaciones fue de 55 minutos aproximadamente.

Para determinar las componentes de velocidad radial y tangencial, se adimensionalizan y discretizan las ecuaciones que definen a la función corriente y a la circulación (9), y se emplean los resultados obtenidos en el sistema de ecuaciones anterior. Las expresiones resultantes en diferencias finitas son

$$
\begin{gathered}
\mathrm{u}_{\mathrm{i}, \mathrm{j}}=\frac{\Psi_{\mathrm{i}, \mathrm{j}-2}-8 \Psi_{\mathrm{i}, \mathrm{j}-1}+8 \Psi_{\mathrm{i}, \mathrm{j}+1}-\Psi_{\mathrm{i}, \mathrm{j}+2}}{12 \Delta \mathrm{r} \Delta \mathrm{zi}} ; \\
\mathrm{v}_{\mathrm{i}, \mathrm{j}}=\frac{\Gamma_{\mathrm{i}, \mathrm{j}}}{\Delta \mathrm{r} \mathrm{i}}
\end{gathered}
$$

Para determinar el flujo volumétrico primario, Qp, que es la cantidad de fluido que fluye alrededor del eje de simetría, el flujo volumétrico secundario, Qs, que es la cantidad de fluido que fluye en el plano r$\mathrm{z}$, y el coeficiente de par, $\mathrm{Cm}$, que es el par requerido para hacer girar el disco, se emplean las siguientes ecuaciones adimensionales (Lang E., et .al., 1994)

$$
\begin{gathered}
\mathrm{Qp}=(\mathrm{H} / \mathrm{E}) \int_{0}^{\mathrm{Z}} \int_{\mathrm{o}}^{\mathrm{r}} \mathrm{v} \mathrm{dr} \mathrm{dz} ; \\
\mathrm{Qs}=2 \pi(\mathrm{H} / \mathrm{E}) \mathrm{r}_{\mathrm{V} . c .}^{\mathrm{r}} \int_{\mathrm{o}}^{\mathrm{z}_{\mathrm{V} . c .}} \mathrm{udz} \\
\mathrm{Cm}=\left.\frac{4 \pi}{(\mathrm{H} / \mathrm{E}) \mathrm{Re}} \int_{0}^{\mathrm{H} / \mathrm{E}} \frac{\partial \mathrm{v}}{\partial \mathrm{z}}\right|_{\mathrm{z}} \mathrm{r}^{2} \mathrm{dr}
\end{gathered}
$$

En los parámetros anteriores, $\mathrm{u}$, es la velocidad radial, $\mathrm{v}$, es la velocidad azimutal del flujo, ( $\mathrm{r}_{\mathrm{vc.c}}, \mathrm{z}_{\mathrm{v} . c .}$ ) son las coordenadas que ubican el centro del vórtice en el plano r-z. La obtención de los flujos volumétricos primario, secundario y del coeficiente de par se determinan al encontrar la función de variación de las componentes de velocidad u y v a través de la coordenada radial y axial, integrarlas, y finalmente evaluarlas a lo largo del radio y altura requeridos por las ecuaciones. Cabe mencionar que el programa se validó al comparar sus resultados con los obtenidos en las referencias (Pao H-P, 1972) y (Bertelá M. y Gori F., 1982), encontrándose buena aproximación. En la Tabla 1 se muestra una comparación de resultados para el valor más alto (en el centro del núcleo) de la función corriente meridional.

Tabla 1. Comparación de resultados para validar el programa.

\begin{tabular}{|l|l|l|l|}
\hline Referencia & Reynolds & H/E & $\Psi$ \\
\hline Pao H-P & 100 & 1 & $-1.12 \times 10^{-2}$ \\
\hline Bertelá M. y Gori F. & 100 & 1 & $-1.20 \times 10^{-2}$ \\
\hline Este trabajo & 100 & 1 & $-1.17 \times 10^{-2}$ \\
\hline
\end{tabular}

Nexo Revista Científica Vol. 28, No. 01, pp. 01-14/Junio 2015 


\section{RESULTADOS Y DISCUSIÓN}

Una vez resuelto el sistema de ecuaciones (11), (12), (13) junto con las condiciones de frontera (10), a través del software diseñado, se hicieron corridas para un número de Reynolds de $3 \times 10^{4}$. Se seleccionó este número de Reynolds debido a que se encuentra en el rango del flujo laminar $(\operatorname{Re}<100,000)$, con ello se asegura la convergencia de las ecuaciones rectoras, tal como se indica en la referencia (Lang E., et al., 1994). Los campos de velocidad radial y tangencial, para los cinco casos a analizar y para un número de Reynolds de $3 \times 10^{4}$, se muestran en las Figs. 2 a 11. Cabe mencionar que los datos que se obtuvieron con el programa fueron tratados con Matlab R2014a para realizar los gráficos. Al analizar la componente de velocidad radial (u), para $\mathrm{Re}=3 \times 10^{4}$ y relación $\mathrm{H} / \mathrm{E}=1$, Fig. 2 , se observan dos flujos, uno en la parte superior que se aleja del centro de rotación hacia la pared vertical, y otro en la parte inferior que se mueve en dirección opuesta al anterior, más una zona de velocidad cero entre ellos. Los valores máximos se ubican en el centro de los flujos, teniendo como magnitudes $3.93 \times 10^{-4}$ y $-2.26 \times 10^{-4}$. El flujo radial positivo es grande en las cercanías de la frontera giratoria y genera un movimiento intenso sobre el disco impulsor. Algo similar, pero con un flujo en dirección contraria, se aprecia en el fondo del recipiente. Ambos flujos producen que entre sus zonas de influencia aparezca una zona donde no existe flujo radial, teniéndose como flujo principal las componentes azimutal y axial. Para las relaciones geométricas (H/E) de $1.25,1.5,1.75$ y 2.0 , Figs. $4,6,8$ y 10 , se observa el mismo comportamiento, es decir, una zona positiva cerca del disco impulsor y otra negativa que abarca el resto del sistema. Este comportamiento es similar al reportado para un $\mathrm{Re}=5 \times 10^{4}$ y relación $\mathrm{H} / \mathrm{E}=1$, según referencia (Lizardi R. A., et al., 2007). Las magnitudes máximas de los flujos positivos y negativos se muestran en la Tabla 2.

Tabla 2. Valores de la componente de velocidad radial en el centro de los flujos.

\begin{tabular}{|l|l|l|}
\hline $\mathbf{H} / \mathbf{E}$ & $\mathbf{u}_{\text {máx. }}$ & $\mathbf{u}_{\text {mín. }}$ \\
\hline 1 & $3.93 \times 10^{-4}$ & $-2.26 \times 10^{-4}$ \\
\hline 1.25 & $4.31 \times 10^{-4}$ & $-1.70 \times 10^{-4}$ \\
\hline 1.5 & $3.93 \times 10^{-4}$ & $-1.08 \times 10^{-4}$ \\
\hline 1.75 & $4.51 \times 10^{-4}$ & $-8.55 \times 10^{-5}$ \\
\hline 2 & $4.25 \times 10^{-4}$ & $-7.76 \times 10^{-5}$ \\
\hline
\end{tabular}

Al analizar la componente de velocidad azimutal (v), para un Reynolds de $3 \times 10^{4}$ y relación $\mathrm{H} / \mathrm{E}=1$, Fig. 3 , se observa que en la zona superior derecha del sistema su valor es el más alto. De allí, su magnitud va disminuyendo, desde la frontera en movimiento hacia el eje del sistema, fondo y pared del recipiente. La componente de velocidad azimutal del fluido tiene un valor grande sobre el disco rotatorio y depende de la coordenada radial y axial. Respecto a la coordenada radial, la velocidad azimutal comienza con un valor de cero sobre en el eje de rotación, posteriormente empieza a crecer su magnitud hasta un máximo, cerca de la frontera rígida, y finalmente disminuye nuevamente su valor hasta hacerse cero sobre la pared del recipiente. El comportamiento en la coordenada axial también tiene una tendencia a disminuir desde el disco impulsor, donde toma su magnitud máxima, hasta el fondo del depósito, donde su valor se hace cero. Para los factores de forma (H/E) de 1.25, 1.5, 1.75 y 2.0, Figs. 5, 7, 9 y 11, se aprecia el mismo comportamiento, es decir, se tiene una velocidad tangencial grande cerca del disco rotatorio y de allí va disminuyendo su magnitud hacia el fondo rígido, eje del sistema y pared del cilindro. Este comportamiento es similar al reportado para un $\mathrm{Re}=5 \times 10^{4}$ y relación $\mathrm{H} / \mathrm{E}=1$, según referencia (Lizardi R. A., et al., 2007). Cabe mencionar que el valor de las líneas de velocidad azimutal constante tienen prácticamente la misma magnitud para todos los casos, lo cual no es de extrañarse pues para este trabajo se manejó una velocidad angular y un radio constantes en todos los sistemas. La diferencia estriba en que al aumentar la relación geométrica, la zona de influencia de la velocidad tangencial es cada vez más baja en las cercanías del fondo del recipiente.

Nexo Revista Científica Vol. 28, No. 01, pp. 01-14/Junio 2015 
A. Lizardi, H. Terres, R. López, M. Vaca, J. Flores, S. Chávez

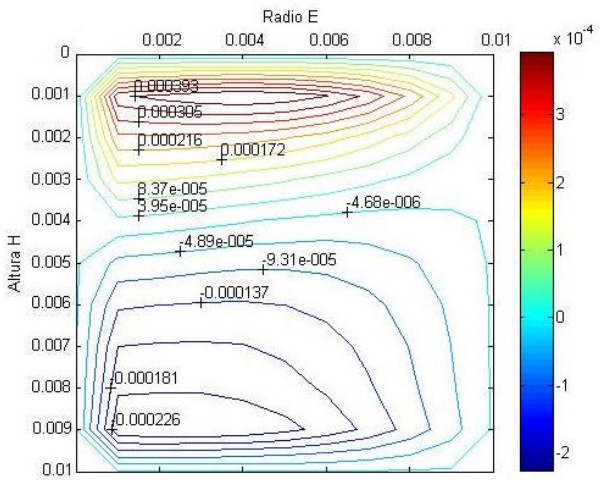

Fig. 2 Velocidad radial (u) para $R e=3 \times 10^{4}$ y factor de forma 1.0

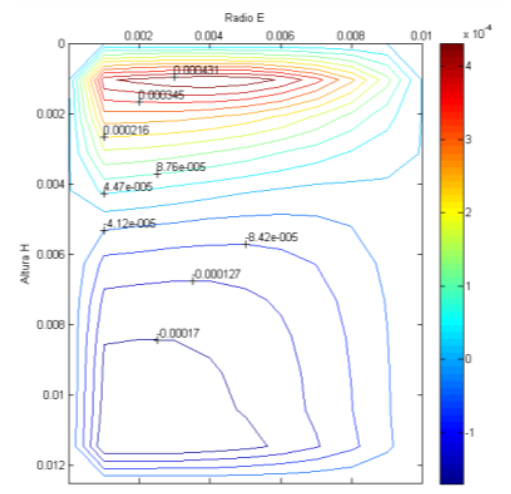

Fig. 4 Velocidad radial (u) para $\operatorname{Re}=3 \times 10^{4}$ y factor de forma 1.25

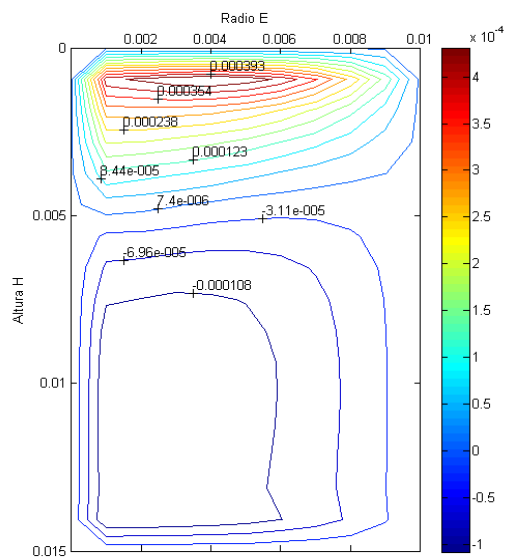

Fig. 6 Velocidad radial (u) para $\operatorname{Re}=3 \times 10^{4} y$ factor de forma 1.5

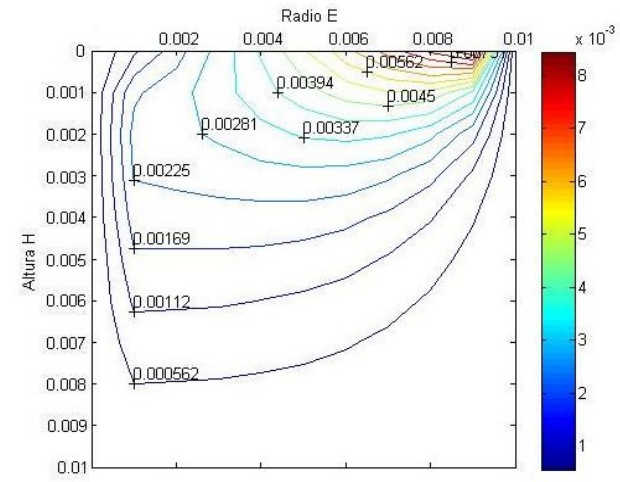

Fig. 3 Velocidad tangencial (v) para $\mathbf{R e}=3 \times 10^{4}$ y factor de forma 1.0

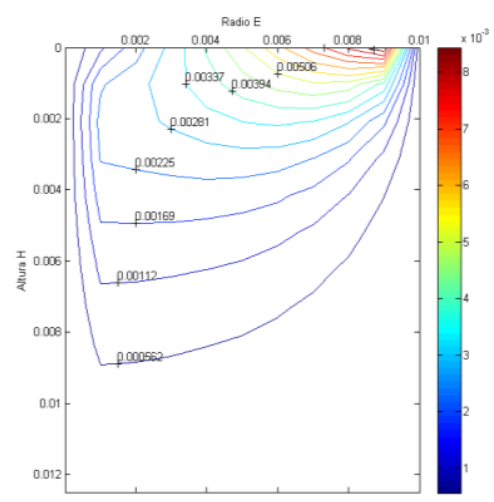

Fig. 5 Velocidad tangencial (v) para $R e=3 \times 10^{4} y$ factor de forma 1.25

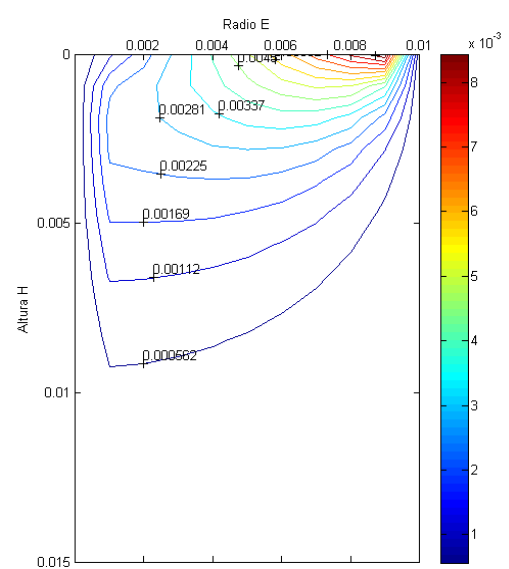

Fig. 7 Velocidad tangencial (v) para $\operatorname{Re}=3 \times 10^{4}$ y factor de forma 1.5 


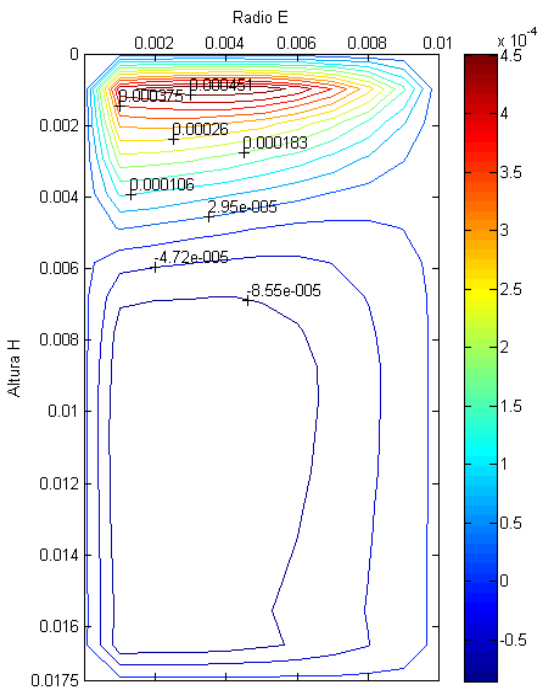

Fig. 8 Velocidad radial (u) para $\operatorname{Re}=3 \times 10^{4}$ y factor de forma 1.75

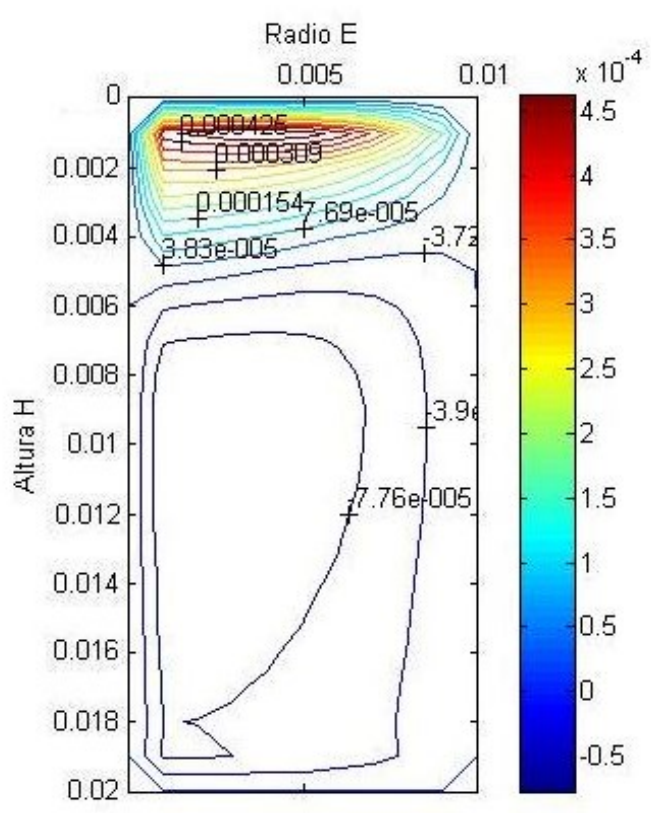

Fig. 10 Velocidad radial (u) para $\operatorname{Re}=3 \times 10^{4}$ y factor de forma 2.0

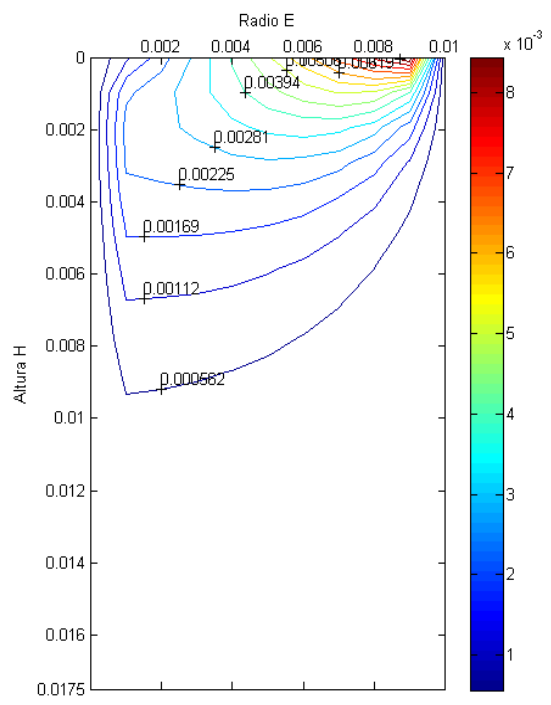

Fig. 9 Velocidad tangencial (v) para $\operatorname{Re}=3 \times 10^{4}$ y factor de forma 1.75

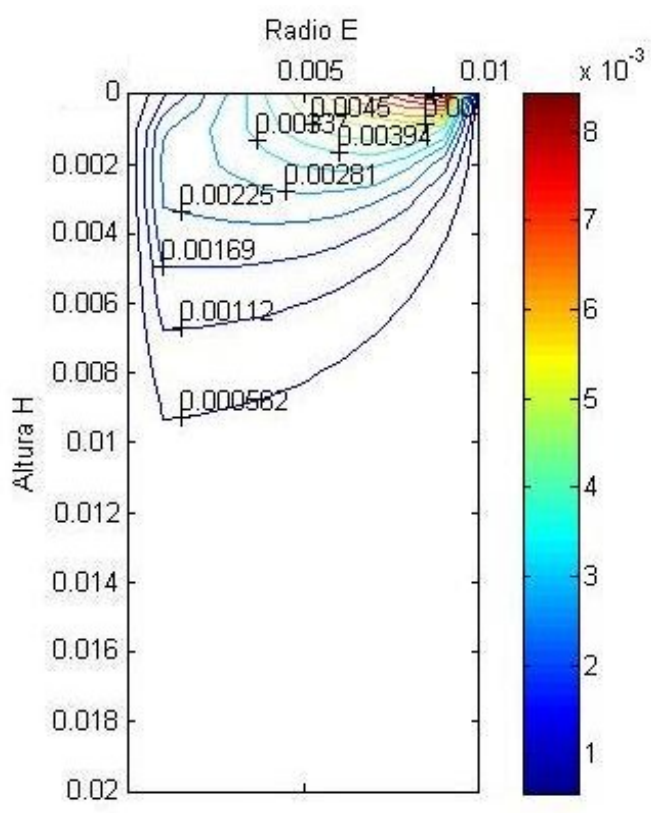

Fig. 11 Velocidad tangencial (v) para $\operatorname{Re}=3 \times 10^{4}$ y factor de forma 2.0

Por otro lado, en la Fig. 12, se muestran los resultados de la componente de velocidad radial (u) para un $\mathrm{Re}=3 \times 10^{4}$, distintas posiciones en la profundidad relativa $\mathrm{z} / \mathrm{H}$, tres ubicaciones del eje radial: $\mathrm{E} / 4=\mathrm{a}$, $\mathrm{E} / 2=\mathrm{b}$ y $3 \mathrm{E} / 4=\mathrm{c}$ y los cinco sistemas a analizar. Para el depósito con factor de forma $\mathrm{H} / \mathrm{E}=1$ y la posición $\mathrm{E} / 4=\mathrm{a}$, se aprecia que la componente de velocidad radial comienza en cero, debido a que sobre el impulsor predomina la velocidad tangencial. Posteriormente se observa la zona donde el flujo se dirige horizontalmente hacia la pared del recipiente, esto se indica por el signo positivo de la velocidad. Se aprecia que el valor de la velocidad radial va aumentando hasta un máximo de $4.07 \times 10^{-4}$ y de allí comienza a disminuir hasta cero, donde a partir de este punto se presenta un cambio del sentido del flujo. Finalmente se observa la zona donde ahora el flujo se dirige hacia el eje del sistema, esto se indica por el signo negativo de la velocidad. En esta parte se aprecia que la velocidad radial va aumentando hasta un máximo negativo de $-2.70 \times 10^{-4}$, y de allí nuevamente disminuye su magnitud hasta llegar a cero cuando se ubica sobre el fondo del cilindro. Para el mismo sistema pero para las posiciones de $\mathrm{E} / 2=\mathrm{b}$ y $3 \mathrm{E} / 4=\mathrm{c}$ se observa el mismo comportamiento pero con valores distintos, en este caso las magnitudes máximas positivas y negativas son de: $4.28 \times 10^{-4} \mathrm{y}-2.39 \times 10^{-4}, 2.51 \times 10^{-4}$ y $-1.22 \times 10^{-4}$, respectivamente. 
$\mathrm{Al}$ analizar los factores de forma de $1.25,1.5,1.75$ y 2.0 se aprecia que el comportamiento es parecido al anterior pero con valores distintos. Las magnitudes máximas positivas y negativas se muestran en la Tabla 3.

Tabla 3. Valores de la componente de velocidad radial en los puntos máximos y mínimos.

\begin{tabular}{|l|l|l|l|}
\hline $\mathbf{H} / \mathbf{E}$ & Posición & $\mathbf{u}_{\text {máx. }}$ & $\mathbf{u}_{\text {mín. }}$ \\
\hline 1 & $\mathrm{E} / 4=\mathrm{a}$ & $4.07 \times 10^{-4}$ & $-2.70 \times 10^{-4}$ \\
\hline 1 & $\mathrm{E} / 2=\mathrm{b}$ & $4.28 \times 10^{-4}$ & $-2.39 \times 10^{-4}$ \\
\hline 1 & $3 \mathrm{E} / 4=\mathrm{c}$ & $2.51 \times 10^{-4}$ & $-1.22 \times 10^{-4}$ \\
\hline 1.25 & $\mathrm{E} / 4=\mathrm{a}$ & $4.49 \times 10^{-4}$ & $-2.15 \times 10^{-4}$ \\
\hline 1.25 & $\mathrm{E} / 2=\mathrm{b}$ & $4.59 \times 10^{-4}$ & $-1.86 \times 10^{-4}$ \\
\hline 1.25 & $3 \mathrm{E} / 4=\mathrm{c}$ & $2.69 \times 10^{-4}$ & $-9.84 \times 10^{-5}$ \\
\hline 1.5 & $\mathrm{E} / 4=\mathrm{a}$ & $4.43 \times 10^{-4}$ & $-1.47 \times 10^{-4}$ \\
\hline 1.5 & $\mathrm{E} / 2=\mathrm{b}$ & $4.64 \times 10^{-4}$ & $-1.25 \times 10^{-4}$ \\
\hline 1.5 & $3 \mathrm{E} / 4=\mathrm{c}$ & $2.69 \times 10^{-4}$ & $-6.44 \times 10^{-5}$ \\
\hline 1.75 & $\mathrm{E} / 4=\mathrm{a}$ & $4.63 \times 10^{-4}$ & $-1.09 \times 10^{-4}$ \\
\hline 1.75 & $\mathrm{E} / 2=\mathrm{b}$ & $4.77 \times 10^{-4}$ & $-9.26 \times 10^{-5}$ \\
\hline 1.75 & $3 \mathrm{E} / 4=\mathrm{c}$ & $2.77 \times 10^{-4}$ & $-4.79 \times 10^{-5}$ \\
\hline 2 & $\mathrm{E} / 4=\mathrm{a}$ & $4.76 \times 10^{-4}$ & $-7.99 \times 10^{-5}$ \\
\hline 2 & $\mathrm{E} / 2=\mathrm{b}$ & $4.88 \times 10^{-4}$ & $-6.78 \times 10^{-5}$ \\
\hline 2 & $3 \mathrm{E} / 4=\mathrm{c}$ & $2.83 \times 10^{-4}$ & $-3.53 \times 10^{-5}$ \\
\hline
\end{tabular}

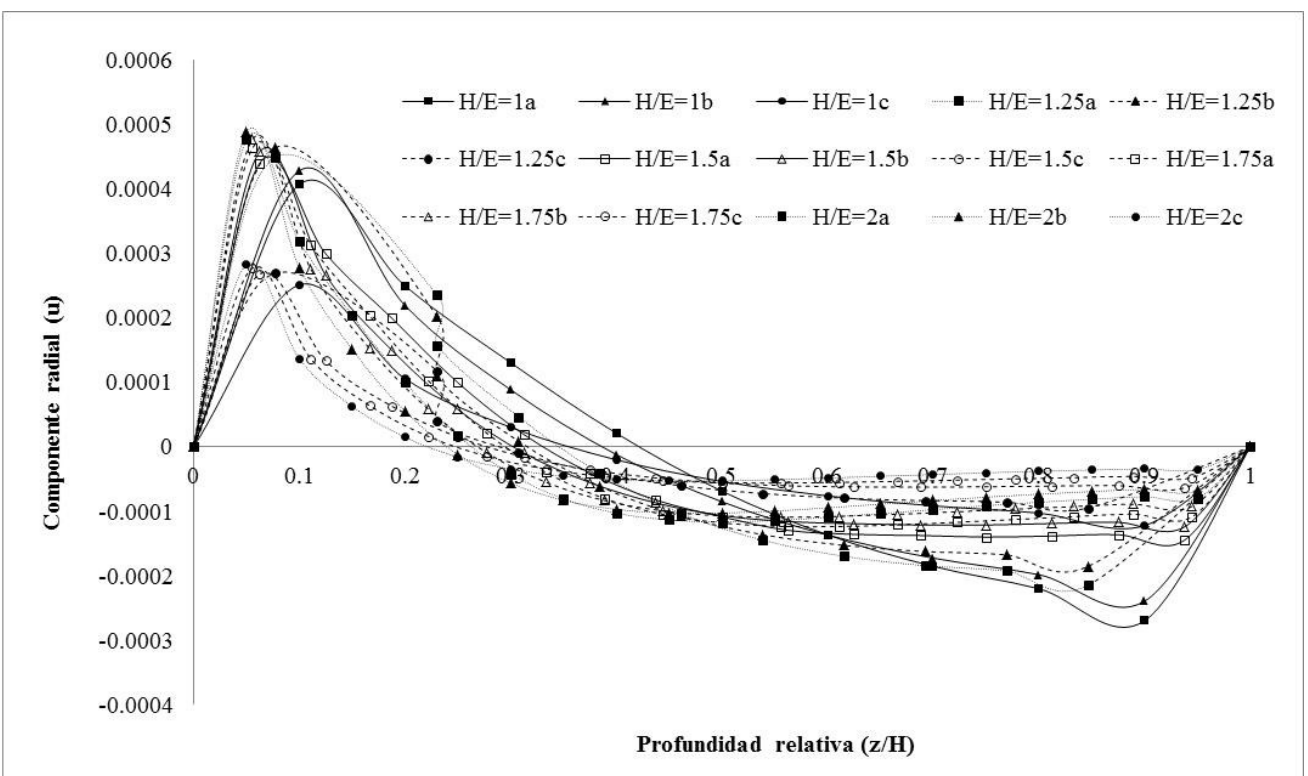

Fig. 12 Componente de velocidad radial (u) para $\mathrm{Re}=3 \times 10^{4}$ en $\mathrm{E} / 4=\mathrm{a}, \mathrm{E} / 2=\mathrm{b}$ y $3 \mathrm{E} / 4=\mathrm{c}$ y $\operatorname{los}$ cinco sistemas

En la Fig. 13, se muestran los resultados de la componente de velocidad azimutal (v) para un $R e=3 \times 10^{4}$, distintas posiciones en el eje $\mathrm{z}$, tres ubicaciones del eje radial: $\mathrm{E} / 4=\mathrm{a}, \mathrm{E} / 2=\mathrm{b}$ y $3 \mathrm{E} / 4=\mathrm{c}$ y los cinco sistemas a analizar. Para el depósito con factor de forma $\mathrm{H} / \mathrm{E}=1$ y la posición $\mathrm{E} / 4=\mathrm{a}$, se aprecia que la componente de velocidad tangencial comienza con un valor de 0.002 sobre el impulsor, posteriormente se presenta un ligero incremento hasta llegar a un máximo y de allí se observa una disminución de su magnitud hasta hacerse cero sobre el fondo del recipiente. Para la posición de $\mathrm{E} / 2=\mathrm{b}$, se aprecia que la componente de velocidad comienza con un valor de 0.005 , posteriormente se presenta una tendencia a disminuir su magnitud a lo largo de la coordenada axial hasta llegar a cero sobre el fondo del depósito. Finalmente, para la posición $3 \mathrm{E} / 4=\mathrm{c}$, se tiene un comportamiento parecido al de la posición antes mencionada pero con un valor sobre el disco rotatorio de 0.008 . Al analizar los factores de forma de $1.25,1.5,1.75$ y 2.0 se aprecia que el comportamiento es igual al del caso anterior, es decir, para las

Nexo Revista Científica Vol. 28, No. 01, pp. 01-14/Junio 2015 
posiciones de $\mathrm{E} / 4=\mathrm{a}, \mathrm{E} / 2=\mathrm{b}$ y $3 \mathrm{E} / 4=\mathrm{c}$ se tiene que la componente de velocidad azimutal inicia con las magnitudes de $0.002,0.005$ y 0.008 , y posteriormente sus valores se hacen cero al llegar a la frontera rígida del fondo del recipiente. Como se mencionó previamente, este comportamiento obedece a que para este trabajo se ha mantenido la velocidad angular y un radio constantes en todos los sistemas y por lo tanto sus magnitudes no cambian. La velocidad tangencial generada por el impulsor solo tiene influencia en una zona de la cámara cilíndrica, acorde a la velocidad angular del disco, después de esa zona su intensidad va disminuyendo en regiones alejadas de la frontera en movimiento.

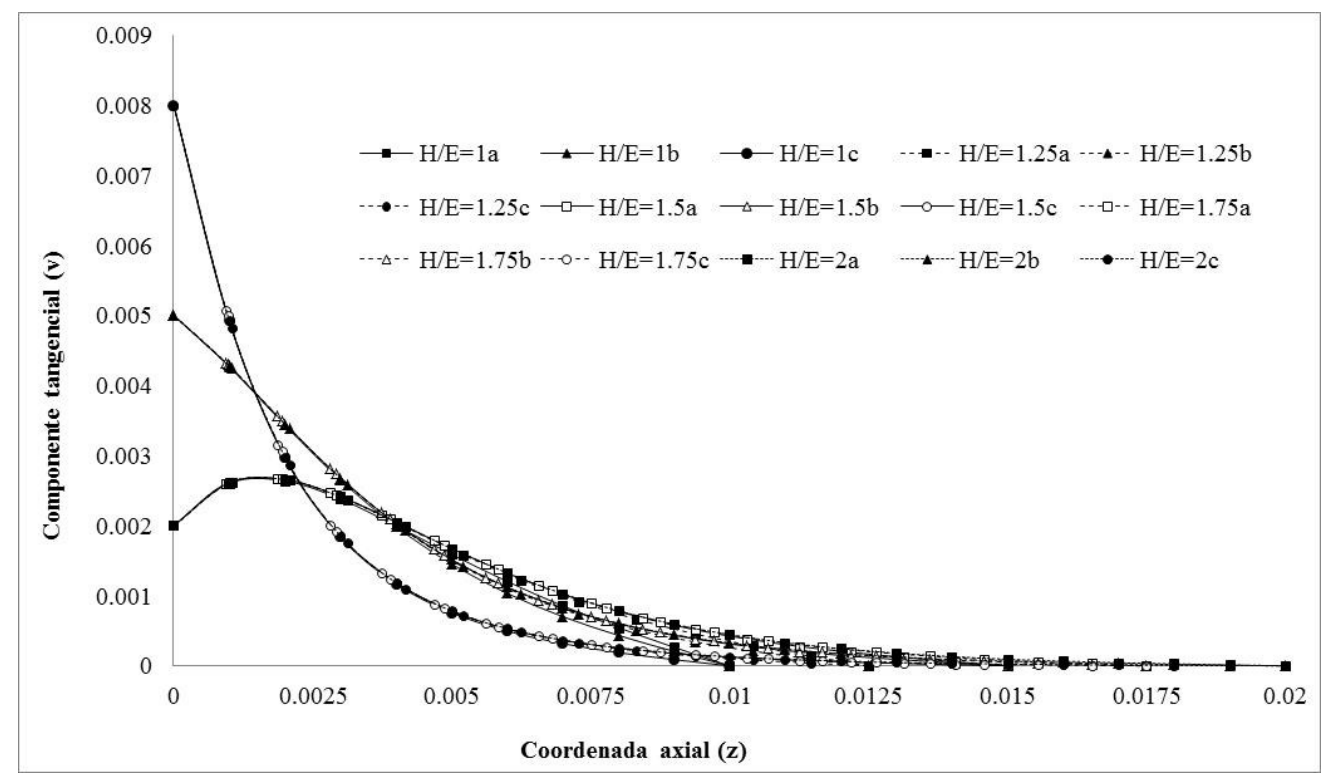

Fig. 13 Componente de velocidad tangencial (v) para $\mathrm{Re}=3 \times 10^{4}$ en $\mathrm{E} / 4=\mathrm{a}, \mathrm{E} / 2=\mathrm{b}$ y $3 \mathrm{E} / 4=\mathrm{c}$ y los cinco sistemas

En la Fig. 14 se muestran los resultados del comportamiento de los flujos volumétricos primario, Qp, (línea continua) y secundario, Qs, (líneas discontinua) a lo largo del eje radial, para un $\mathrm{Re}=3 \times 104$, y los cinco sistemas a analizar. Para el depósito con factor de forma $\mathrm{H} / \mathrm{E}=1$, se observa que el flujo volumétrico primario $(\mathrm{Qp})$ comienza con un valor de cero sobre el eje de rotación, posteriormente su magnitud se va incrementando hasta llegar a un máximo de 1.63×10-9, en la región cercana a la pared del recipiente, y de allí comienza a disminuir nuevamente hasta hacerse cero sobre la frontera rígida del sistema. Al analizar los factores de forma de $1.25,1.5,1.75$ y 2.0 se aprecia que el comportamiento es parecido al del caso anterior, pero con valores distintos. Sus magnitudes se muestran en la Tabla 4. Al observar los valores numéricos se aprecia que el flujo volumétrico primario se va incrementando al aumentar la altura del depósito lo cual corresponde con la descripción física del problema.

Tabla 4. Valores máximos del flujo volumétrico primario (Qp) y secundario (Qs).

\begin{tabular}{|l|l|l|l|}
\hline $\mathbf{H} / \mathbf{E}$ & Qp $_{\text {máx. }}$ & Qs $_{\text {máx. }}$ & Qs $_{\text {mín. }}$ \\
\hline 1 & $1.63 \times 10^{-9}$ & $1.48 \times 10^{-7}$ & $-3.03 \times 10^{-7}$ \\
\hline 1.25 & $3.07 \times 10^{-9}$ & $2.50 \times 10^{-7}$ & $-2.86 \times 10^{-7}$ \\
\hline 1.5 & $3.23 \times 10^{-9}$ & $4.31 \times 10^{-7}$ & $-1.50 \times 10^{-7}$ \\
\hline 1.75 & $3.43 \times 10^{-9}$ & $4.72 \times 10^{-7}$ & $-1.34 \times 10^{-7}$ \\
\hline 2 & $4.78 \times 10^{-9}$ & $4.98 \times 10^{-7}$ & $-1.19 \times 10^{-7}$ \\
\hline
\end{tabular}




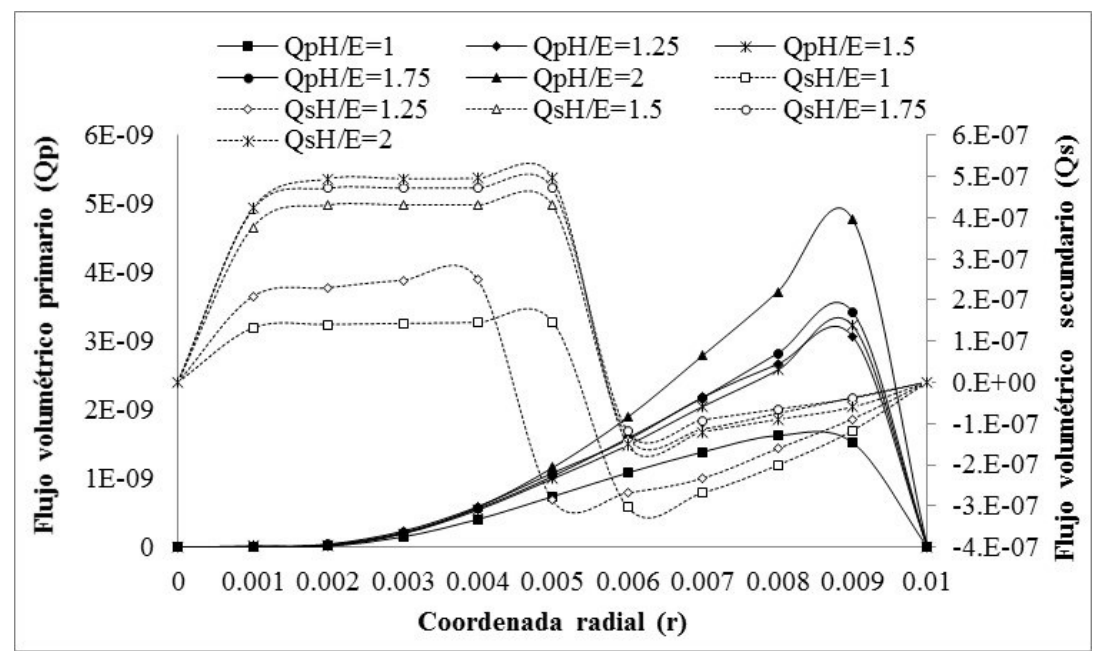

Fig. 14 Flujos volumétricos primario, Qp, (línea continua) y secundario, Qs, (línea discontinua) para $\operatorname{Re}=3 \times 10^{4}$ y los cinco sistemas

Por otro lado, al analizar el flujo volumétrico secundario (Qs) para el depósito con factor de forma $\mathrm{H} / \mathrm{E}=1$, se aprecia que su valor comienza con cero sobre el eje de rotación, posteriormente el flujo se dirige verticalmente hacia la frontera superior, incrementando su magnitud hasta llegar a un máximo de $1.48 \times 10^{-7} \mathrm{y}$ de allí comienza a disminuir hasta llegar a cero, donde hay un cambio en el sentido del flujo, dirigiéndose éste hacia el fondo del recipiente. El valor máximo negativo que alcanza el flujo volumétrico secundario es de $-3.03 \times 10^{-7}$. Finalmente su magnitud se hace nuevamente cero al llegar a la pared rígida del sistema. Este comportamiento concuerda con la descripción física del flujo secundario donde el fluido se mueve rotacionalmente en el plano r-z, generando un flujo vertical ascendente de lado izquierdo y descendente de lado derecho, ambos respecto al centro del vórtice. Al analizar los factores de forma de 1.25, 1.5, 1.75 y 2.0 se observa que el comportamiento es parecido al del caso anterior, pero con valores distintos. Las magnitudes máximas y mínimas se muestran en la Tabla 4 . Al igual que en el flujo volumétrico primario, se aprecia que el flujo secundario se va incrementando al aumentar la altura del depósito.

Tabla 5. Valores máximos del coeficiente de par (Cm).

\begin{tabular}{|l|l|}
\hline $\mathbf{H} / \mathbf{E}$ & $\mathbf{C m}_{\text {máx. }}$ \\
\hline 1 & $1.61 \times 10^{-12}$ \\
\hline 1.25 & $1.88 \times 10^{-12}$ \\
\hline 1.5 & $2.20 \times 10^{-12}$ \\
\hline 1.75 & $2.59 \times 10^{-12}$ \\
\hline 2 & $3.13 \times 10^{-12}$ \\
\hline
\end{tabular}

En la Fig. 15 se muestran los resultados del comportamiento del coeficiente de par para un $R e=3 \times 10^{4}$ y los cinco sistemas a analizar. En todos los casos se observa un comportamiento similar, comienzan con un valor pequeño cerca del eje de rotación y de allí su magnitud se va incrementando ligeramente hasta llegar a un máximo en la región cercana a la pared del recipiente. Los valores más altos que presentan los factores de forma 1.0, 1.25, 1.5, 1.75 y 2.0, se muestran en la Tabla 5. En la Fig. 15 también se aprecia que la variación del coeficiente de par es pequeña a lo largo de la coordinada radial y se mantiene prácticamente constante para todas las relaciones geométricas analizadas. Lo anterior es debido a que en este trabajo el diámetro del impulsor y la velocidad angular permanecen constantes. La magnitud del coeficiente de par es pequeña debido a que en su ecuación rectora (15) se encuentra en el denominador el número de Reynolds, que en este estudio tiene un valor alto. 


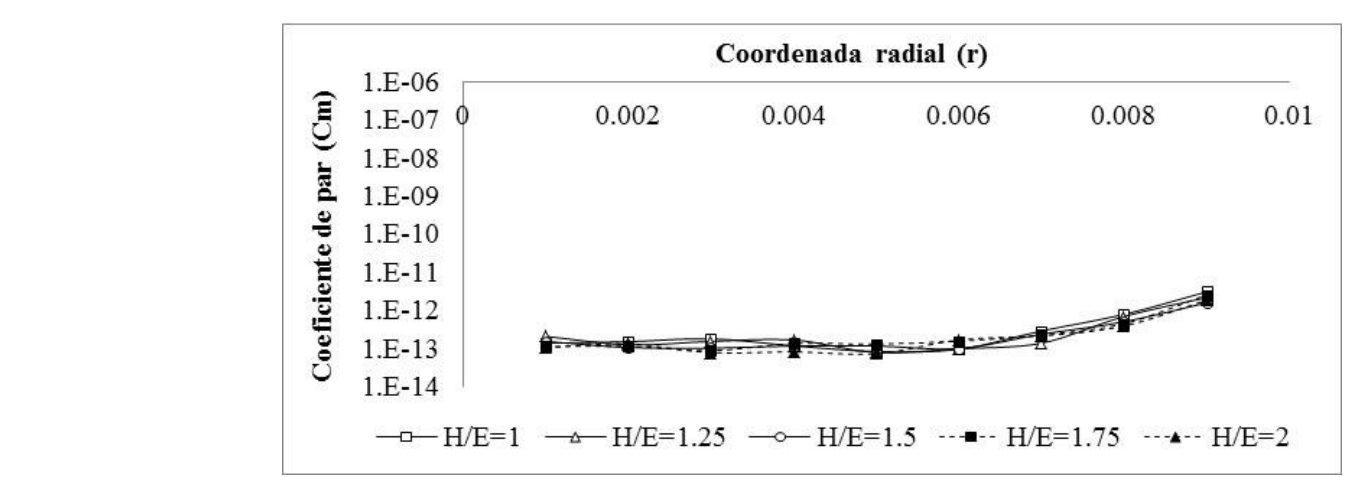

Fig. 15 Coeficiente de par $(\mathrm{Cm})$ para $\mathrm{Re}=3 \times 10^{4}$

\section{CONCLUSIONES}

Analizando los resultados anteriores y tomando como referencia la relación geométrica de 1.0 y comparándolo con los factores de forma de $1.25,1.5,1.75$ y 2.0 , se encontró que: la componente de velocidad radial $(u)$ se incrementó en su valor máximo positivo en un $7.24 \%, 8.41 \%, 11.45 \%$ y 14.02 $\%$, respectivamente; la componente de velocidad tangencial (v) mantuvo los mismos valores máximos en todas las relaciones geométricas, el flujo volumétrico primario aumentó un $88.34 \%, 98.16 \%, 110.43$ $\%$ y $193.25 \%$, respectivamente; el flujo volumétrico secundario se incrementó $68.92 \%, 191.22 \%$, $218.92 \%$ y $236.48 \%$, respectivamente; y el coeficiente de par aumentó un $16.77 \%$, $36.64 \%, 60.87 \%$ y $94.41 \%$, respectivamente. Los resultados anteriores revelan que al modificar la relación de esbeltez del sistema las propiedades cinemáticas estudiadas no siguen un patrón de variación homogéneo y que dependerá del interés que se tenga en alguna de ellas para

\section{NOTACIÓN}

$\mathrm{g}$

$\mathrm{r}$

u

$\mathrm{V}$

W

Z

$\mathrm{E}$

$\mathrm{H} \quad$ Altura del depósito (m)

$\mathrm{Cm}$ Coeficiente de par adimensional

Qp Flujo volumétrico primario adimensional

Qs Flujo volumétrico secundario adimensional

P Presión $[\mathrm{Pa}]$

$\mathrm{R} \quad$ Coordenada radial dimensional [m]

Re Número de Reynolds

U Componente de velocidad radial $[\mathrm{m} / \mathrm{s}]$
V Componente de velocidad tangencial $[\mathrm{m} / \mathrm{s}]$

W Componente de velocidad axial $[\mathrm{m} / \mathrm{s}]$

$\mathrm{Z} \quad$ Coordenada axial dimensional [m]

$\Delta \mathrm{r} \quad$ Espacio de la malla en dirección radial

$\Delta z \quad$ Espacio de la malla en dirección axial

$\gamma$ Función circulación $\left[\mathrm{m}^{2} / \mathrm{s}\right]$

$\varsigma \quad$ Función vorticidad [1/s]

$\psi \quad$ Función corriente meridional $\left[\mathrm{m}^{3} / \mathrm{s}\right]$

$v$ Viscosidad cinemática $\left[\mathrm{m}^{2} / \mathrm{s}\right]$

$\rho \quad$ Densidad $\left[\mathrm{kg} / \mathrm{m}^{3}\right]$

$\varepsilon \quad$ Error relativo

$\sigma \quad$ Paso iterativo del programa

$\Phi$ Coordenada tangencial dimensional

$\Omega \quad$ Velocidad angular del impulsor [ $\mathrm{rad} / \mathrm{s}]$

$\Gamma \quad$ Función circulación adimensional

$\xi \quad$ Función vorticidad adimensional

$\Psi \quad$ Función corriente adimensional

\section{REFERENCIAS}

Bertelá M., Gori F. (1982). Laminar flow in a cylindrical container with a rotating cover. Journal of fluids engineering. 104 (1):31-39. doi:10.1115/1.3240849.

Gerber N. (1975). Properties of rigidly rotating liquids in closed partially filled cylinders. ASME, Transactions, Series E - Journal of Applied Mechanics. 42:734,735. 
Khalili A., Adabala R. R. (1995). Flow induced by a asymmetrically placed disk rotating coaxially inside a cylindrical casing. Acta Mechanica. Vol 113.

Landau y Lisfshitz. (1982) Fluids Mechanics. Vol. 6, Pergamon Press.

Lang E., Sridhar K., Wilson N. W. (1994). Computational study of disk driven rotating flow in a cylindrical enclosure. Journal of fluids engineering. 116 (4):815-820.

Lizardi R. A., Díaz C. A., López C. R., J. R. Morales G., Lara V. A., Terres P. H., Portillo V. J. de J. (2007). Análisis experimental y numérico del flujo rotatorio en un medio finito. The Mexican Journal of Electromechanical Engineering. Vol. 11, No. 4, pp. 199-205. ISSN 1665-0654.

Lizardi, A., López, R., Morales, J. R., Terres, H., Lara A., Hernández, A. (2010) Análisis numérico de las funciones corriente, circulación y vorticidad, para flujo rotatorio con frontera rígida. Revista internacional de métodos numéricos para cálculo y diseño de ingeniería. Vol. 26, número 3, pp. 179185.

Lizardi, A., Terres, H., López, R., Zavala, C. N., Lara, A., Morales, J. R. (2013). Análisis numérico de la velocidad radial y axial para flujo rotatorio con distintos factores geométricos y números de Reynolds. VI Congreso Internacional de Métodos Numéricos en Ingeniería. Morelia, Michoacán, México.

Pao H-P. (1972). Numerical solution of the Navier Stokes equations for flows in the disk-cylinder system. Phys. Fluids. 15 (1):4-11.

Sturzenegger J. C., Sarasúa L. G., Martí A. C. (2012). Analytical solutions for the axisymmetric flow inside a cylindrical container with a rod along the axis at low Reynolds numbers. Journal of Fluids and Structures. Vol. 28, pp. 473-479.

Yu P., Lee T. S., Zeng Y., Low H. T. (2007). Characterization of flow behavior in an enclosed cylinder with a partially rotating end wall. Physics of fluids. 19, 057104, doi: 10.1063/1.2731420.

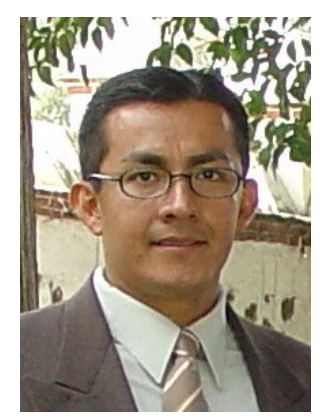

Arturo Lizardi Ramos. Ingeniero Mecánico ESIME-IPN (1995). Maestro en Ciencias en Ingeniería Mecánica SEPI ESIME-IPN (1997). Profesor Investigador de la UAM-A, México. Áreas de interés: Análisis experi-mental y numérico de problemas en Termofluidos. 